MedieKultur | Journal of media and communication research | ISSN 1901-9726

Article - Theme section

\title{
Datafication and the Push for Ubiquitous Listening in Music Streaming
}

\section{Rasmus Rex Pedersen}

MedieKultur 2020, 69, 71-89

Published by SMID | Society of Media researchers In Denmark | www.smid.dk The online version of this text can be found open access at www.mediekultur.dk

\begin{abstract}
This article discusses Spotify's approach to music recommendation as datafication of listening. It discusses the hybrid types of music recommendation that Spotify presents to users. The article explores how datafication is connected to Spotify's push for the personalization and contextualization of music recommendations based on a combination of the cultural knowledge found in editorial curation and the potential for large-scale personalization found in algorithmic curation. The article draws on the concept of ubiquitous music and other understandings of the affective and functional aspects of music listening as an everyday practice to reflect upon how Spotify's approach to datafication of listening potentially leads it to prioritize music recommendations that entice users to engage in inattentive and continuous listening. In extension to this, the article seeks to contribute with knowledge about how the datafication of listening potentially shapes listening practices and conceptions of relevance and quality in music recommendation.
\end{abstract}

\section{Keywords}

Music streaming, algorithmic curation, Spotify, datafication 


\section{Introduction}

Spotify has developed significantly since its launch a little over a decade ago. From initially focusing on the technologic challenges of (frictionless) music distribution, Spotify is increasingly focusing on delivering personal music experiences. Spotify's approach to delivering such music experiences has been based on a "curatorial turn" (Eriksson, Fleischer, Johansson, Snickars, \& Vonderau, 2019), delivering a combination of personal and contextual music recommendations.

This shift accommodates the multitude of different listening practices that listeners engage in in their daily lives. For example, playlists sorted into what Spotify calls "Genres \& Moods" provide an alternative to the lean-back listening traditionally offered by radio by expanding the range to include activities such as workouts, focus and gaming, as well as introducing personalized recommendations, sometimes in the form of algotorial recommendations that combine algorithmic and editorial approaches (Bonini \& Gandini, 2019).

The small but rapidly expanding base of literature that deals with the datafication of listening has to a large extent focused on technical, social or business-oriented perspectives on the subject. The datafication of listening is critiqued as a surveillance technology that serves to consolidate the economic and cultural power of a few technology companies providing music streaming as part of a platform economy (Drott, 2018a; Hesmondhalgh, 2019; Prey, 2016, 2018). The extensive amount of data provided by music streaming has also been analysed as a factor in changing music business dynamics and decision-making processes (Maasø \& Hagen, 2019; Morgan, 2020; O'Dair \& Fry, 2020), and as a source of internal competition and differentiation among music streaming services (Morris \& Powers, 2015). In addition to this, some scholars have focused on how the datafication of listening leads to new curatorial practices and potentially different types of algorithmic bias (Besseny, 2020; Morris, 2015; Werner, 2020).

Based on the existing literature on the datafication of listening, this article seeks to reflect on how Spotify uses data to construct specific implied listeners, and how these datafied notions of listening potentially shape how users explore, experience and interact with music - and thereby also the ontology and epistemology of music listening.

Theoretically, this article draws primarily on the literature from media and communication studies as outlined above. In addition, it develops conceptions of "ubiquitous listening" (Kassabian, 2013a, p. 61) along with other affective and functional perspectives on music listening in order to provide a framework for understanding different listening practices accommodated by streaming services. The concept of ubiquitous listening is explored in dialogue with a study of Spotify's curatorial turn, discussing how the service's turn to personalized and contextualized music recommendations based on datafication implies an emphasis on data on user behaviour collected primarily through implicit feedback, and how these data can be shaping future recommendations (Prey, 2016, 2018). In extension to this, the article discusses how the datafication of listening promotes concep- 
tions of the quality and relevance of music recommendations that potentially promote continuous and inattentive listening.

Drawing on the conception of music recommendation as a subjectivation process (Drott, 2018b), the article argues that data-driven curatorial practices shape conceptions of relevance of music recommendations in ways that amplify and encourage user practices of ubiquitous listening.

\section{Ubiquitous music and ubiquitous listening}

The concept of ubiquitous listening was introduced by Anahid Kassabian to describe "the listening that fills our days [...]. How do we listen to the music we hear, and how does that listening engage us and activate the world we move in?" (Kassabian, 2013a, p. xi). Although Kassabian and her colleagues argue that the concept defies a simple definition (Garcia Quinones et al., 2016), the basic premise is that even though music is often listened to without primary attention, it still produces affective responses that lead to emotions (Kassabian, 2013a, p. xi).

In this sense, Kassabian focuses on music listening as an everyday practice. She notes that despite the many ways we encounter music in our everyday lives without actively engaging with it, conceptions of listening tend to leave out inattentive listening to focus on attentive listening. This bias towards attentive listening can, for instance, be found in music scholarship, where the ideal listener is attentive and proficiently trained; it may seem to be rooted not only in ideological and aesthetic judgements, but also methodological challenges. Studying music is in itself an attentive process, which might explain the preference for studying attentive listening. Studying ubiquitous music is much more complex: "If we mean to study music that is listened to inattentively, we cannot ask people about it, because the process is not a conscious one" (Kassabian, 2013b, p. 90).

Ubiquitous music is closely related to the ideas of background music, elevator music or muzak. These terms used to be assigned to music produced or adapted specifically to function as background music, whereas music by original artists was conceived as foreground music. All three terms carry clear functional and commercial connotations. Muzak was originally a trademark of the company of the same name that transcended its origin to become a tag for a mix of musical styles and functions. In line with this, scholars have often understood background music, elevator music and muzak as examples of music as a mass cultural form that is fundamentally different from more serious or learned music (see, for example, Attali, 1985), and the terms 'muzak' and 'elevator music' often carry connotations of boring, vapid or even cheesy music. Moreover, scholarly literature on the subject often focuses on the use of instrumental music as background music (see, for example, Lanza, 2004).

Kassabian builds on some of the literature that has engaged with these concepts related to background listening (e.g. Lanza, 2004; Sterne, 1997; Stockfelt, 1997). She notes 
that since the 1980s, the music used as background music has increasingly become music by original artists (Kassabian, 2013a, p. 5). Kassabian argues that what characterizes ubiquitous music has more to do with modes of listening than with musical characteristics. She defines ubiquitous listening as

\begin{abstract}
a mode of listening dissociated from specific generic characteristics of the music. In this mode, we listen 'alongside', or simultaneously with, other activities. [...] it relies on a kind of 'sourcelessness'. Whereas we are accustomed to thinking of most musics, and most cultural products, in terms of authorship and location, this music comes from the plants and the walls and, potentially, our clothes. It comes from everywhere and nowhere. Its projection looks to erase its production as much as possible, posing instead as a quality of the environment (Kassabian, 2013a, pp. 9-10).
\end{abstract}

And because music has become embedded in the environment, and listening therefore has become ubiquitous, listening has become a "less-than-fully-attentive activity" (Kassabian, 2013a, p. 51), as most people rarely listen to music as a primary focus. Instead, listening to music has become something that accompanies other simultaneous activities in the attentional field.

As noted above, ubiquitous listening is a concept concerned more with modes of listening than the aesthetic qualities of the music. Any kind of music can, in principle, be ubiquitous music (even if some music demands its listener's attention to a degree that makes it a less probable candidate), and there are therefore no specific genre traits or musical qualities that characterize the music. This also means that it is hard to pin down. One person's ubiquitous music might be another person's favourite foreground music. The same song might even be used by the same person as the object of active listening and appreciation in the morning and function as ubiquitous music when it accompanies a workout in the gym in the afternoon. When ubiquitous listening is defined by the mode of listening, then the only way of identifying it is by understanding the listening context. Kassabian links ubiquitous listening to the branding practices of, for example, cafes and clothing stores, and understanding where the music is being played-in terms of both the place and the medium-is a decent (but not perfect) indicator of how the music is being listened to.

Kassabian's conception of ubiquitous music could, however, be more thorough in developing an understanding of how the music that we encounter through less-thanfully-attentive listening affects listeners, beyond merely producing affective responses and emotions. From a psychological and sociological perspective, the issue of inattentive listening has been addressed by scholars like DeNora (1999) and Sloboda (Juslin \& Sloboda, 2001; Sloboda, 2010). Central to the work of these scholars is the consideration of the relationship between music listening (even if non-attentive) and self-identity. DeNora argues for an understanding of self-identity as connected to the ongoing activity of individuals rather than a fixed essence; she focuses-as apparent in the title "Music as a technology of the self"-on the agency of listeners. Importantly, she emphasizes that music can be 
seen as reflexively constituting emotional states in people rather than being an expression of internal emotional states (DeNora, 1999, p. 57).

However, the increased access and portability offered by first iPods and later smartphones is leading to changing listening practices. Firstly, as Michael Bull writes about the iPod, "The use of these technologies appears to bind the disparate threads of much urban movement together, both 'filling' the spaces 'in-between' communication or meetings and structuring the spaces thus occupied" (Bull, 2005, p. 344). Bull further argues that iPods blur the distinction between work and leisure, as well as between 'the 'non-spaces' of urban culture and the meaningful spaces associated with any individual's personal narrative" (Bull, 2005, p. 347). Secondly, iPods amplify the increasing tendency towards individualized media consumption (Bull, 2005; Livingstone, 2002).

With the advent of digital music services - and music streaming in particular-we have seen a convergence of listening practices which extends beyond mobility and individualization: different music media imply particular listening practices. However, streaming services like Spotify represent a convergence of a wide range of implied listening practices. Let me offer an example: through curated playlists, music streaming lends itself to the lean-back ubiquitous listening also offered by radio; but it lends itself equally well to the kind of music exploration and discovery previously offered through 'crate-digging' and listening in the listening booth at the local record shop. The medium of music streaming implies a multitude of different listening practices and thereby notably extends the logics of mobility and individualization outlined by Bull and Livingstone beyond merely listening to the listener's own curated library of music on the home stereo or the iPod.

This has implications for how music streaming services present music to their users. What constitutes a relevant recommendation changes constantly as we move from situation to situation. As the former chief musicologist at Pandora and architect behind the Music Genome Project phrases it:

\footnotetext{
... the music we listen to each day is a complex amalgam of voluntary and involuntary encounters, and is aligned with the many diverse contexts and circumstances of our daily lives: practical (motivation at the gym), functional (getting to work), environmental (at the grocery store), social (at the restaurant), and leisure (choosing a CD at home), among others. As such, our 'musical taste' is continually being engaged and queried as we pursue our day-to-day routines and responsibilities, whether we're consciously aware of it or not (Gasser, 2019, p. 4).
}

The idea of music listening as a practice embedded in other social practices has obviously not emerged with the advent of streaming. But even though we should acknowledge that music is also valuable when used as a cultural resource that enriches everyday practices, Hesmondhalgh (2013, p. 40) has argued that we need a sense of constrained agency: although we are able to reflect on our use of music and make use of it to obtain certain objectives, we are still limited by social and psychological dynamics. The datafication of 
listening practices-and the presentation of music and implied listening practices that follow from it-can be understood as such social and psychological dynamics, and the next part of the article explores this further.

To sum things up, the idea of ubiquitous music can be developed to cover three distinct aspects: 1) music listening as a mundane everyday practice, which is often non-attentive; 2) music listening as a reflexive process that uses music as a resource for identity work; and 3) the constant availability of music in the digital age, which enables individualistic listening practices across social situations. In other words, ubiquitous music is closely related to the practice of creating a personal soundtrack for our lives.

\section{Datafication of listening}

Music streaming services have changed significantly over the last decade. From primarily being distribution technologies focused on providing frictionless access to all music everywhere-the proverbial celestial jukebox - the focus has gradually changed to that of providing musical experiences for users (Eriksson et al., 2019). In order to do this, streaming services like Spotify and Pandora rely heavily on the datafication of listening.

The datafication of listening, and the real time data feedback loop that it creates, is arguably the most important distinction between streaming services and previous music media. Collecting and analysing metadata about music and users' interaction with it enables streaming services to provide each user with personalized recommendations. The assumption that lies behind this is that "the more accurately a streaming service is able to zero in on the tastes of the individual listener, the more time the listener will spend on a service" (Prey, 2016, p. 32). In this sense, the datafication of listening marks a shift in the structuring logic of music recommendation: where the music industry used to be focused on persuading listeners to buy a new release, streaming services are now trying to predict what users want or need (Harvey, 2014; Prey, 2016).

The datafication of listening that happens in music streaming services like Spotify is in line with the datafication taking place across media and communications platforms, where it has become a "legitimate means to access, understand and monitor people's behavior" (van Dijck, 2014, p. 198)[italics in original]. However, as van Dijck argues, the ideological basis of datafication relies on problematic ontological and epistemological claims based on a widespread, albeit not necessarily precise, belief in the objectiveness and precision of the quantification and tracking of human behaviour and sociality (Finn, 2017; van Dijck, 2014).

Apart from utilizing datafication as a means of providing personalized recommendations for users, streaming services also treat data as an important source of value in itself. As Eric Drott writes: "Even as data drives the various features and functionalities platforms offer to users, most notably those relating to the customization of the listen- 
ing experience, such data is also capable of being monetized in a variety of ways" (Drott, 2018a, p. 237). He lists three of these ways: 1) As a commodity, where data about users are sold directly to third parties in advertising, finance or data aggregation; 2) as a factor of production, where data are used to define the users and sell their attention to advertisers, specifying their demographic and psychographic attributes; and 3) as an asset, where user data contribute to the market valuation of a platform and add value to a potential capital investment or acquisition (ibid.). Because many of us use music as a 'technology of the self'-using music to shape, reflect, or process emotions and social situations-information about the music we listen to also offers potential insights into the listeners' innermost selves. In this sense, the datafication of listening, depending on the perspective we take, provides an ideal tracking technology or a powerful surveillance technology (Drott, 2018a, p. 233).

The datafication of listening is therefore dialectically related to ubiquitous music. On the one hand, datafication is a way of providing music recommendations that fit with the individualistic and reflexive listening practices associated with ubiquitous listening, while on the other hand, it actively shapes listening practices.

\section{Hybrid approaches to music recommendation}

In order to understand the relationship between datafication and listening practices, it is relevant to have a basic understanding of how music recommendation works at Spotify. Like most platforms, Spotify employs multiple approaches to recommendation. The user's path to the next track might be through searching, filtering, featured playlists and releases, or automated recommendations on the basis of the music just listened to. Data play a crucial role in all of these approaches, but the role played varies significantly from approach to approach.

Focusing solely on the way data are employed to optimize the user experience (thereby leaving out the secondary value of data mentioned in the paragraph above), three main categories can be identified. Data are used as 1) the basis for algorithmic curation; 2) in collaboration with editorial curation, both as ways to identify relevant tracks for editors to consider for inclusion on playlists, and for further algorithmic individualization of the editorial playlist-Spotify refers to this approach as "algotorial" (Dredge, 2018); and 3) for strategic purposes: data about users' interactions with the platform are used as a basis for decisions about the interface design as well as priorities in editorial recommendations.

Data for these purposes can be gathered in different ways, and the general characteristic of the many different data sources that Spotify uses is that they are built for scalability. In 2014, Spotify acquired the music analytics company The Echo Nest. The Echo Nest's approach to music analytics is characterized by a combination of automated (primarily machine learning-based) content analysis of the music, semantic analysis of metadata 
about the music, and analysis of users' interactions with the platform (Prey, 2018). This means that the data that serve as a basis for recommendation and interface design can be collected across all users and all tracks at any given time.

Platforms often collect these types of data using combinations of explicit and implicit feedback. Explicit feedback collected, for example, through tracking devices such as 'like' buttons and reviews has become a central part of the data flows of online platforms (Gerlitz \& Helmond, 2013), and is often viewed as a more precise indication of user preferences. However, the understanding of musical similarity that Spotify bases its recommendations on is tied to a conviction that musical similarity derives more from "cultural meaning" than from audio signals (Chodos, 2019, p. 48). This has prompted Spotify and The Echo Nest to opt to build primarily on implicit feedback based on users' actions rather than explicit feedback based on their stated preferences. This approach sidesteps the issue of getting users to indicate their preferences on a large scale while simultaneously focusing on data from implicit feedback and using that as a basis for interpretation of the social situation and cultural meaning of listening. But the choice also comes with its disadvantages. The fact that a user listens to a specific track tells us little about why, and knowing how many times a user listens to that track gives only a crude indication of the user's affinity for the specific track.

At Spotify, datafication of listening is closely connected to what has been called a "curatorial turn" (Eriksson et al., 2019, p. 61). After initially focusing on the technology of music distribution as the core of its business, Spotify has, since 2013, shifted its strategic focus increasingly towards providing unique music-related experiences (Eriksson et al., 2019, p. 67), leading to a strategy that focuses on personalized and contextual music recommendations.

In the interface design of the Spotify application, the homepage acts as a gateway to content that tries to meet these strategic goals. Playlists have become a primary tool for music discovery and have become an increasingly important source of listening. Data on how users engage with Spotify are notoriously hard to get access to, but in the prospectus released when filing for an IPO in 2018, Spotify gave small insights into the share of listening that playlists represent:

\footnotetext{
Many of our Users also rely on Spotify to help soundtrack their day, through editoriallycurated playlists like RapCaviar or personalized machine-generated playlists like Discover Weekly, Daily Mix, or Release Radar. We now program approximately $31 \%$ of all listening on Spotify across these and other playlists, compared to less than $20 \%$ two years ago (Spotify Technology S. A., 2018, p. 98).
}

Although users spend considerably more time listening to their own personal playlistsapproximately 36 pct. of monthly content hours (Spotify Technology S.A., 2018, p. 108) the rapidly increasing share of curated and algorithmic playlists reflect how Spotify's strategic focus on music recommendation shapes user behaviours. This strategic effort is 
Article: Datafication and the Push for Ubiquitous Listening in Music Streaming

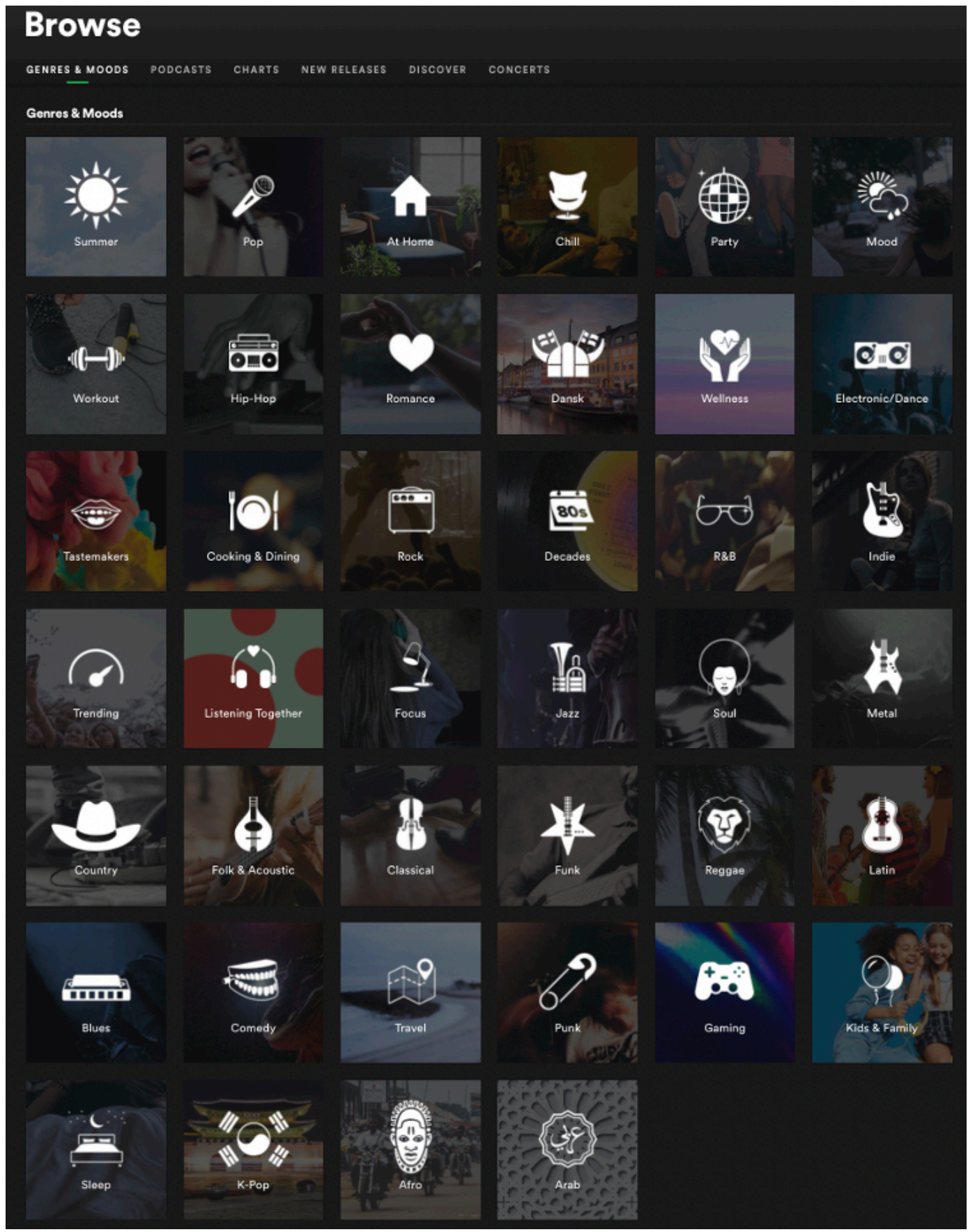

Figure 1: Screenshot of the 'Browse' tab from Spotify's desktop application for Mac

reflected in the affordances of the interface design. The user is predominantly presented with content algorithmically or editorially curated by Spotify and leaves only little room for the user's own searches and homemade playlists (for an in-depth analysis of this, see Besseny, 2020). 
As indicated in the quotations above, Spotify strives to provide music recommendations that take account of situational context as well as personal taste. Although the two approaches are often combined, the analysis will focus firstly on how Spotify uses editorial playlists to invite users to situational listening, and then turn to its use of data to create algorithmically curated personal playlists. The reason for this is that each perspective adds to the understanding of the relationship between ubiquitous listening and the datafication of listening.

\section{Genre and mood: Spotify's use of editorial playlists}

With Spotify's strategic agenda in mind, it is no surprise that a vast majority of the playlists featured in Spotify's Moods and Genres-92 pct., according to one music industry analytics company (Joven, 2018) - are curated by Spotify themselves.

In practice, the coupling of moods and genres under the 'Browse' tab in Spotify's application interface represents an integration of what have traditionally been treated as separate approaches to categorizing music listening as related to either the musical content or the situational context of listening. Spotify's approach to music recommendation acknowledges that both are important, and provides inroads into its playlists that cater for both.

As seen in Figure 1, Spotify places musical genres (Pop, Hip-Hop, Electronic/Dance, Rock, R\&B, Indie, Jazz, Soul, Metal, Country, Folk \& Acoustic, Classical, Funk, Reggae, Latin, Blues, Punk, K-Pop, Afro) alongside moods and activities (At Home, Chill, Party, Mood, Workout, Romance, Wellness, Cook \& Dining, Listening Together, Focus, Travel, Gaming, Sleep). These are supplemented by labels that relate to other categorizations such as demographics (Dansk [Danish], Arab, Kids \& Family), seasons (Summer, Christmas, etc.), relative popularity (Trending, Tastemakers), and time of release (Decades).

Under each of these labels, the user is presented with playlists that fit that overall label. The playlists that are visible to the user are influenced by that user's previous listening, and include algorithmically curated personal playlists as well as both local and international editorial playlists.

The playlists are named in ways that indicate musical style, but also often invite the user to engage with the playlist in particular ways. Some are named by the simple characterization of popularity or a time period (Today's Top Hits, Global Top 50, All Out 90s); some clearly designate a musical genre (RapCaviar, Rock Classics, Are \& Be, ¡Viva Latino!); some designate suggested listening situations (Songs to Sing in the Shower, Power Workout, Your Favorite Coffeehouse, Top Gaming Tracks); and others invite users to use the music as a tool for emotional work (Mood Booster, Have a Great Day!, Confidence Boost, Broken Heart).

A study by music analytics company Chartmetric showed in 2018 that though the majority of Spotify's playlists (57 pct.) were content-based, the median number of followers was higher for context-based playlists (Joven, 2018). The distinction between moods 
Article: Datafication and the Push for Ubiquitous Listening in Music Streaming

\begin{tabular}{|c|c|c|c|c|}
\hline Position & Curator & Followers & Playlist Name & Genre/Mood \\
\hline 1 & Spotify & 26655210 & Today's Top Hits & Pop, top lists \\
\hline 2 & $\begin{array}{l}\text { Spotify } \\
\text { Charts }\end{array}$ & 15457150 & Global Top 50 & Pop, top lists \\
\hline 3 & Spotify & 13320675 & RapCaviar & Rap, top lists, hip-hop \\
\hline 4 & Spotify & 10622784 & ¡Viva Latino! & Latin \\
\hline 5 & Spotify & 10163879 & Baila Reggaeton & Latin \\
\hline 6 & Spotify & 9522773 & Songs to Sing in the Car & Pop, travel \\
\hline 7 & Spotify & 8413670 & All Out 00s & Pop, dance pop, throwback \\
\hline 8 & Spotify & 8046806 & Rock Classics & Rock, classic rock \\
\hline 9 & Spotify & 7463637 & All Out $80 \mathrm{~s}$ & $\begin{array}{l}\text { Soft rock, new wave pop, throwback, } \\
\text { pop }\end{array}$ \\
\hline 10 & Spotify & 7367499 & Beast Mode & $\begin{array}{l}\text { Electronic dance music (EDM), electro } \\
\text { house }\end{array}$ \\
\hline 11 & Spotify & 5975131 & Get Turnt & Rap, hip-hop \\
\hline 12 & Spotify & 5961671 & Peaceful Piano & Focus, sleep, compositional ambient \\
\hline 13 & Spotify & 5944734 & All Out 90s & Dance pop, throwback, pop \\
\hline 14 & Spotify & 5877856 & Chill Hits & Pop \\
\hline 15 & Spotify & 5865380 & Hot Country & $\begin{array}{l}\text { Top lists, country, contemporary coun- } \\
\text { try, country road }\end{array}$ \\
\hline 16 & Spotify & 5609473 & Mint & $\begin{array}{l}\text { Pop, EDM, top lists, tropical house, } \\
\text { electronic/dance }\end{array}$ \\
\hline 17 & Spotify & 5607006 & Songs to Sing in the Shower & Pop, at home \\
\hline 18 & Spotify & 5472582 & Happy Hits! & Pop, mood \\
\hline 19 & Spotify & 5435721 & Mood Booster & Pop, mood \\
\hline 20 & Spotify & 5283753 & Are \& Be & $\mathrm{R} \& \mathrm{~B}, \mathrm{pop}$, top lists \\
\hline 21 & Spotify & 5254223 & Esquenta Sertanejo & Sertanejo universitario, sertanejo pop \\
\hline 22 & Spotify & 5094221 & Motivation Mix & EDM, workout, tropical house \\
\hline 23 & Spotify & 5087098 & Hit Rewind & Pop \\
\hline 24 & Spotify & 4980459 & I Love My'90s Hip-Hop & $\begin{array}{l}\text { Rap, hip hop, hip-hop, black history is } \\
\text { now }\end{array}$ \\
\hline 25 & Spotify & 4977110 & Top Brasil & Sertanejo universitario, funk carioca \\
\hline 26 & Spotify & 4719113 & Have a Great Day! & Rock, mood, pop rock \\
\hline 27 & Spotify & 4708902 & All Out $10 \mathrm{~s}$ & Pop \\
\hline 28 & Spotify & 4625078 & Mega Hit Mix & Pop \\
\hline 29 & Spotify & 4548113 & Rock This & Top lists, rock, modern rock \\
\hline 30 & Spotify & 4525933 & This Is Ed Sheeran & Pop \\
\hline 31 & Spotify & 4252794 & Soft Pop Hits & Pop \\
\hline 32 & Spotify & 4202276 & Dance Party & $\begin{array}{l}\text { EDM, house, party, electro house, } \\
\text { electronic/dance }\end{array}$ \\
\hline 33 & Spotify & 3978679 & Teen Party & Pop \\
\hline 34 & Spotify & 3921771 & Sleep & Sleep, compositional ambient, focus \\
\hline 35 & Spotify & 3908389 & Workout & Pop, workout \\
\hline 36 & Spotify & 3898346 & Verano Forever & Latin, party, summer \\
\hline 37 & Spotify & 3852174 & New Music Friday & Pop, pop rap \\
\hline
\end{tabular}


Article: Datafication and the Push for Ubiquitous Listening in Music Streaming

\begin{tabular}{|c|c|c|c|c|}
\hline 38 & Spotify & 3851707 & 90s' Rock Anthems & Rock, alternative rock, throwback \\
\hline 39 & Spotify & 3818727 & Top Hits Philippines & Pop \\
\hline 40 & Spotify & 3787818 & Rock en Español & $\begin{array}{l}\text { Latin rock, rock en español, Latin } \\
\text { alternative }\end{array}$ \\
\hline 41 & Spotify & 3783299 & Your Favorite Coffeehouse & $\begin{array}{l}\text { Mood, folk-pop, indie folk, new Ameri- } \\
\text { cana, folk \& acoustic }\end{array}$ \\
\hline 42 & Spotify & 3782021 & All Out 70 s & $\begin{array}{l}\text { Mellow gold, soft rock, throwback, } \\
\text { rock }\end{array}$ \\
\hline 43 & Spotify & 3713883 & Relax \& Unwind & $\begin{array}{l}\text { Chill, folk-pop, indie folk, chamber pop, } \\
\text { folk \& acoustic }\end{array}$ \\
\hline 44 & Spotify & 3696338 & Baladas Románticas & Latin pop, Latin \\
\hline 45 & Spotify & 3640909 & Acoustic Covers & $\begin{array}{l}\text { Chill, neo mellow, viral pop, channel } \\
\text { pop, folk \& acoustic }\end{array}$ \\
\hline 46 & Spotify & 3613131 & Funk Hits & Funk carioca \\
\hline 47 & Spotify & 3569081 & Éxitos México & Latin \\
\hline 48 & Spotify & 3479009 & Brain Food & $\begin{array}{l}\text { EDM, focus, electronic/dance, elec- } \\
\text { tronic }\end{array}$ \\
\hline 49 & $\begin{array}{l}\text { Chilled } \\
\text { Cow }\end{array}$ & 3410168 & $\begin{array}{l}\text { Lofi Hip Hop Music - Beats } \\
\text { To Relax/Study To }\end{array}$ & Chillhop, lo-fi beats \\
\hline 50 & Spotify & 3398872 & $\begin{array}{l}\text { Acoustic Hits: Oldies but } \\
\text { Goodies }\end{array}$ & Pop, folk-pop, neo mellow, throwback \\
\hline
\end{tabular}

Figure 2. The 50 playlists with most followers on Spotify

Source: Chartmetric.com (27 June 2020)

and genres might suggest a dichotomy between playlists curated based on the stylistic features of the musical content and playlists curated based on the intended listening situations. However, the study also showed that a growing share of playlists was categorized as hybrid-that is, Spotify tags the playlists with both content- and context-related tags, enabling users to arrive at the particular playlist through click paths that indicate interest for either a particular musical genre or music suited for a particular context (Joven, 2018). Figure 2 lists the most popular playlists on Spotify and the tags associated with them.

If we return for a moment to Kassabian's categorization of ubiquitous music as the music that fills our days and is listened to without primary attention, we see that Spotify's ambition to "help [users] soundtrack their day" (Spotify Technology S.A., 2018, p. 98) has implications for the role music plays in these users' lives. By encouraging users to engage in music listening alongside or simultaneously with everyday situations and activities like driving the car, cooking, dining, studying, working out or even sleeping, Spotify gently pushes users towards treating music as a quality of the situational environment. This focus on the ways music can be used to shape, reinforce or change emotions is even more clearly pronounced in the mood playlists that are not directly associated with particular activities or situations, but rather associated with a particular frame of mind. Users are encouraged to use music to provide an energy boost, improve work productivity, or increase focus and concentration (Eriksson \& Johansson, 2017, p. 74). Playlists with titles 
such as Feelin' Good, Life Sucks, Sad Songs, or Couples in Love invite listeners to use music strategically to produce affective responses that shape emotions.

\section{Made for you: Algorithmic curation and personalization}

Like the editorial playlists, Spotify's emphasis on personal recommendations seems to be the result of a deliberate strategy. Since the acquisition of the music analytics company The Echo Nest in 2013, Spotify has gradually increased its emphasis on algorithmically curated personal recommendations for each user. The Discover Weekly playlist-a personal playlist for each of Spotify's more than 100 million users, updated on a weekly basis-launched as a flagship of this development in 2015. Since then, personal recommendations have gradually become a more prominent aspect of the Spotify homepage. The analysis here will focus on the personal recommendations afforded on the 'Home' and 'Browse' pages of the Spotify application; however, it is important to note that algorithmic recommendations are also found in other places, such as the 'Autoplay' feature, which continues to play similar songs after the user's own choice of music ends, or in the recommended songs that appear when a new playlist is created.

The personal recommendations on the homepage are algorithmically generated for the individual user based on the "algorithmic identity" (Cheney-Lippold, 2011, p. 165) or "musical identity" (Prey, 2018, p. 6) constructed by Spotify. These recommendations fall into three general categories: 1) personal reactivations of the user's favourites (music that has been played recently, or music that has been in heavy rotation); 2) personal recommendations curated for the user (playlists that curate previously listened to music and present it alongside known and unknown music that is similar, or playlists that encourage music discovery, like Discover Weekly or Release Radar); and 3) recommendation of editorial playlists that fit the user's taste profile.

The last category might not seem like personal recommendations; however, an increasing number of editorial playlists are personalized. Based on data pulled from Chartmetric.com in mid-June 2020, 51 of the 100 most popular editorial playlists (based on number of followers) were personalized, meaning that they function as algotorial playlists that adapt an editorial playlist to fit the taste of the specific user. In this sense, Spotify's playlist curation is characterized by an attempt to create a hybrid approach that combines the qualitative musical and cultural knowledge of its playlist editors with the potential for personalization offered by data-driven algorithmic recommendations.

\section{From datafication of listening to ubiquitous listening}

As outlined at the beginning of this article, Eric Drott (2018a, p. 237) argues that the data derived from music streaming platforms like Spotify can be monetized as a commodity, as a factor of production, and as an asset. This monetization is in itself an important aspect of the datafication of listening that mirrors the dynamics found in other platform economies. However, as argued above, Spotify is increasingly pursuing a hybrid approach 
to the datafication of listening in which data are used to provide knowledge about the musical content, the users, the users' musical tastes, and how these tastes are intricately related to the social and emotional practices of listening.

Spotify's data on users can be monetized as a commodity by selling it to third parties, and although data about demographics and musical taste can be valuable in itself, the value is multiplied when linked to specific activities or emotional states. As an example of this, the Bank of England has reportedly been experimenting with Spotify data as a way to provide insights into consumers' sentiments (Marston, 2018). Similarly, data about the frequency of a user listening to running and workout playlists might give an indication of the particular user's health and lifestyle.

As a factor of production, Drott (2018a) argues that data can be used to define users and monetize their attention by selling advertisements on the platform. This is the case with Spotify, but equally it is the combination of contextual music curation and databased personalization that creates a feedback loop where data on listening direct developers to prioritize certain affordances, which in turn shapes listening practices. This is obviously the case with the implementation of personal recommendations where data about users' past listening patterns are used as a basis for future recommendations. But the feedback loop also manifests itself when, for example, data indicate that contextbased playlists have a higher median number of followers than content-based playlists (as found in the Chartmetric study mentioned earlier in this article). This might lead Spotify to put greater emphasis on contextual playlists, thereby inviting users to do more contextbased listening.

Drott (2018a) also argues that data can be monetized as an asset that contributes to a company's market valuation and makes it more attractive for capital investment. Although this aspect is less important for the scope of this article, it is worth noting that Spotify repeatedly accentuated the centrality of data to its business model in the prospectus released before the IPO on Wall Street in 2018 (Spotify Technology S.A., 2018). Furthermore, through data visualizations and big data graphs, Spotify has used data storytelling as an integral part of its appeals for financial investment (Vonderau, 2019, p. 7).

The driving force behind the datafication of listening is an intention to create more engaged users. It is worth noting that engagement can be understood in both quantitative and qualitative terms. It can be understood qualitatively in relation to the level of attention that a listener allocates to the music, as in Kassabian (2013a). However, the data Spotify collects are not suitable for estimating this kind of engagement. Rather, for personal recommendations, it relies on a quantitative measure that understands engagement in relation to time spent on the platform.

In this sense, datafication leads to a potential shift in emphasis from attentive to inattentive listening. Kassabian (2013a) argues that music analysis and recommendation have traditionally had a bias towards attentive listening, to a certain extent because these are themselves acts of attentive listening. With Spotify's approach to music recommenda- 
tion, the perspective shifts, and a particular emphasis is placed on playlists that encourage inattentive listening (Pelly, 2017; Prey, 2019); and even though we should be careful not to assume that design choices determine actual use, Spotify, like all technologies, has affordances that constrain some behaviours and facilitate others (Prey, 2019).

The tendency outlined above is amplified by the fact that Spotify relies so heavily on the datafication of listening as a foundation for both music recommendation and strategic design decisions. The reliance on datafication for these purposes leads to the substitution of qualitative understandings of aesthetic value and musical culture with quantitative measures of engagement and context. This leads to a situation where the bias shifts towards quantitative criteria, thereby potentially creating a bias towards inattentive (background) listening.

We tend to understand recommendation algorithms as neutral and unbiased, but in reality, the algorithms behind automated and personalized culture recommendations are more like sprawling assemblages encompassing computational processes for collecting data and analysing them using statistical calculations to provide recommended actions. The interfaces generally reflect little of the cultural processing behind them (Finn, 2017, p. 16).

Crucially, these algorithms enact theoretical ideas through pragmatic instructions, and the gap between the two is bridged by a 'good-enough' rationalism that prioritizes speed and scalability. This has a significant impact on our culture and everyday life, because these approximations are based on compromises and tend to efface what they do not comprehend (Finn, 2017, p. 22).

As Drott argued, streaming services like Spotify construct a normative listener. Through recommendations, Spotify hails users to use the service in such a way that it can be seen as a subjectification process (Drott, 2018b). Spotify takes a listening approach to datafication in which the normative listener, as suggested by Drott, is a listener for whom music discovery and engagement with the platform offer mutually reinforcement. By measuring engagement primarily by means of quantitative data from implicit feedback from users - such as time spent on the platform, skip rates, listening patterns and playlist creation-Spotify also potentially ends up prioritizing a specific form of music discovery that prompts users to spend more time listening and expend less effort choosing what to listen to next. When Spotify invites users to use the platform to 'soundtrack their day' with a combination of contextual and personal playlists, it is doing exactly this. However, if we follow Drott's (2018b) suggestion and understand music recommendation as a subjectification process, we must also be aware that the normative listener that Spotify constructs is a listener that engages in ubiquitous listening.

The potential implications of this integration of datafication and contextual music recommendations reach beyond the listening practices of individual Spotify users. Datafication and the use of metrics have become increasingly central to decision-making processes, not only internally within streaming services like Spotify, but for stakeholders across the music industry. This way, datafication creates a feedback loop between the 
recommendation and production of music in which "datafication itself may create trends and influence the music culture thanks to the algorithmic affordances of [music streaming services]" (Maasø \& Hagen, 2019, p. 12). The potential implications of this are that data feedback loops create situations where music is treated more as media content that is meant to fit with the platform's agenda for attracting and retaining users than as an artform in itself.

\section{Conclusion}

Since the "curatorial turn" (Eriksson et al., 2019, p. 61) in 2013, Spotify has strategically increased its emphasis on music recommendations that fit the tastes, moods and moments of the user. In doing this, Spotify constructs normative listener profiles and curates playlists that fit these types of listening. These playlists often encourage users to treat music as part of the situational environment and encourage listening practices where music is used to shape, reinforce or process moods and emotions. In this sense, music recommendation can be understood as a subjectification process.

This article has drawn together knowledge on the datafication of listening and Spotify's music recommendation practices and psychological and sociological understandings of musical meaning and reflexive identity in everyday listening practices. By combining the two perspectives, this article seeks to open a discussion on how the datafied notions of listening shape listening practices.

The datafication of listening is an important factor in understanding the strategic motives behind Spotify's approach to music recommendation. Even if Spotify promotes the use of data as something that is meant to optimize the musical experience of usersand, as an extension of this, as a differentiating factor in comparison with its competitors-data are also something that can be monetized directly and indirectly. Spotify has increasingly developed hybrid approaches to music recommendation, combining musical genre and mood/activity in the theme of curated playlists, and combining the cultural knowledge of editorial curation with the potential for personalization of algorithmic curation, in order to provide better a music experience for its users.

By integrating understanding of the datafication of listening at Spotify with theories of everyday listening practices - in particular the notion of ubiquitous listening - this article explores the dialectical relationship between the two. On the one hand, Spotify's approach can be understood as a shift that acknowledges music listening as something mundane and employs datafication to enable listeners to create a personal soundtrack for their day. This implies an emphasis on agency and individualism wherein Spotify treats music as a "technology of the self" (DeNora, 1999). However, as Hesmondhalgh (2013, p. 40) argues, this agency must be understood as constrained by social and psychological dynamics. So, on the other hand, the user agency offered in Spotify's interface design and 
datafied approach to music recommendation can be understood as constrained in ways that potentially shape how users listen and what music they listen to.

The dialectical relationship between ubiquitous listening and datafication has implications for music and music listening. In Spotify's curational turn, both ubiquitous listening and datafication are employed with the aim of providing better a listening experience for users. However, since the datafication of listening relies on quantifiable data to measure the relevance and quality of musical recommendations, there is a risk of ignoring qualitative characteristics of music and musical taste, thereby overcompensating for the previous bias towards attentive listening and instead creating a bias towards inattentive listening. As argued previously in this article, such listening practices can definitely reflect user agency and be a part of the identity work of the individual listener. However, the datafication of listening also implicates focusing on music's functional value as a resource, rather than music's aesthetic value and the depth of the emotions it produces as an object of contemplation and attentive listening.

\section{Notes}

1 Kassabian derives the concept from the notion of ubiquitous computing, and is particularly inspired by ubiquitous computing's potential of embedding computing power into everyday objects, thereby enabling a much more widespread use of computers while simultaneously making our interactions with computers less obvious and attentive.

\section{References}

Attali, J. (1985). Noise: the political economy of music. Minneapolis: University of Minnesota Press.

Besseny, A. (2020). Lost in spotify: folksonomy and wayfinding functions in spotify's interface and companion apps. Popular Communication, 18(1), 1-17. https://doi.org/10.1080/15405702.2019.1701674

Bonini, T., \& Gandini, A. (2019). "First Week Is Editorial, Second Week Is Algorithmic": Platform Gatekeepers and the Platformization of Music Curation. Social Media and Society, 5(4). https://doi. org/10.1177/2056305119880006

Bull, M. (2005). No dead air! The iPod and the culture of mobile listening. Leisure Studies, 24(4), 343-355. https://doi.org/10.1080/0261436052000330447

Cheney-Lippold, J. (2011). A New Algorithmic Identity: Soft Biopolitics and the Modulation of Control. Theory, Culture \& Society, 28(6), 164-181. https://doi.org/10.1177/0263276411424420

Chodos, A.T. (2019). What Does Music Mean to Spotify? An Essay on Musical Significance in the Era of Digital Curation. INSAM Journal of Contemporary Music, Art and Technology, I(2), 36-64.

DeNora, T. (1999). Music as a technology of the self. Poetics, 27(1), 31-56. https://doi.org/10.1016/S0304422X(99)00017-0

Dredge, S. (2018). 10 key talking points from the Spotify investor day today. Retrieved January 8, 2020, from https://musically.com/2018/03/15/talking-points-spotify-investor-day/

Drott, E.A. (2018a). Music as a technology of surveillance. Journal of the Society for American Music, 12(3), 233-267. https://doi.org/10.1017/S1752196318000196 
Drott, E.A. (2018b). Why the Next Song Matters: Streaming, Recommendation, Scarcity. Twentieth-Century Music, 15(3), 325-357. https://doi.org/10.1017/S1478572218000245

Eriksson, M., Fleischer, R., Johansson, A., Snickars, P., \& Vonderau, P. (2019). Spotify Teardown: Inside the Black Box of Streaming Music. Cambridge, Massachusetts: MIT Press. https://doi.org/10.7551/mitpress/10932.001.0001

Eriksson, M., \& Johansson, A. (2017). “Keep Smiling!” : Time, Functionality and Intimacy in Spotify's Featured Playlists. Cultural Analysis, 16(1), 67.

Finn, E. (2017). What Algorithms Want: Imagination in the Age of Computing. Cambridge, Mass.: MIT Press.

Garcia Quinones, M., Kassabian, A., \& Boschi, E. (2016). Ubiquitous Musics - The Everyday Sounds That We Don't Always Notice. (M. Garcia Quinones, A. Kassabian, \& E. Boschi, Eds.). London: Routledge. https:// doi.org/10.1353/tech.2014.0090

Gasser, N. (2019). Why you like it: the science and culture of musical taste. New York: Flatiron Books.

Gerlitz, C., \& Helmond, A. (2013). The like economy: Social buttons and the data-intensive web. New Media and Society, 15(8), 1348-1365. https://doi.org/10.1177/1461444812472322

Harvey, E. (2014). Station to station: The past, present, and future of streaming music. Retrieved from http://pitchfork.com/features/cover-story/reader/streaming/

Hesmondhalgh, D. (2013). Why Music Matters. Chichester: John Wiley \& Sons. Retrieved from https:// ebookcentral.proquest.com/lib/kcl/reader.action?doclD $=1245463$

Hesmondhalgh, D. (2019). The Cultural Industries (4th ed.). London: SAGE Publications.

Joven, J. (2018). Spotify: The Rise of the Contextual Playlist. Retrieved June 20, 2020, from https://blog.chartmetric.com/spotify-the-rise-of-the-contextual-playlist/?gi=edca5db9bafb

Juslin, P.N., \& Sloboda, J.A. (Eds.). (2001). Music and Emotion: Theory and Research. Oxford: Oxford University Press.

Kassabian, A. (2013a). Ubiquitous Listening: Affect, Attention, and Distributed Subjectivity. University of California Press. https://doi.org/10.1525/california/9780520275157.003.0001

Kassabian, A. (2013b). You Say Invisible, I Say Ubiquitous: A (Formally Former) Student's Response to Philip Tagg's 'Caught on the Back Foot: Epistemic Inertia And Visible Music.' Journal of the International Association for the Study of Popular Music, 3(2), 86-95. Retrieved from http://iaspmjournal.net/ index.php/IASPM_Journal/article/view/622/pdf, https://doi.org/10.5429/2079-3871(2013)v3i2.7en

Lanza, J. (2004). Elevator Music. Ann Arbor, MI: University of Michigan Press. https://doi.org/10.3998/mpub.8718

Livingstone, S. (2002). Young People and the Media. London: Sage.

Maasø, A., \& Hagen, A.N. (2019). Metrics and decisions-making in music streaming. Popular Communication. https://doi.org/10.1080/15405702.2019.1701675

Marston, R. (2018, May 1). Spotify playlists in Bank of England's sights. BBC News. Retrieved from https:// www.bbc.com/news/business-43960998

Morgan, B.A. (2020). Revenue, access, and engagement via the in-house curated Spotify playlist in Australia. Popular Communication, 18(1), 32-47. https://doi.org/10.1080/15405702.2019.1649678

Morris, J.W. (2015). Curation by code: Infomediaries and the data mining of taste. European Journal of Cultural Studies, 18(4-5), 446-463. https://doi.org/10.1177/1367549415577387

Morris, J.W., \& Powers, D. (2015). Control, curation and musical experience in streaming music services. Creative Industries Journal, 8(2), 106-122. https://doi.org/10.1080/17510694.2015.1090222

O'Dair, M., \& Fry, A. (2020). Beyond the black box in music streaming: the impact of recommendation systems upon artists. Popular Communication, 18(1), 65-77. https://doi.org/10.1080/15405702.2019.16 27548 
Pelly, L. (2017, December). The Problem with Muzak: Spotify's bid to remodel an industry. Retrieved from https://thebaffler.com/salvos/the-problem-with-muzak-pelly?src=longreads

Prey, R. (2016). Musica Analytica: The Datafication of Listening Robert Prey University of Groningen, Netherlands. In R. Nowak \& A. Whelan (Eds.), Networked Music Cultures (pp. 31-48). Palgrave Macmillan. https://doi.org/10.1057/978-1-137-58290-4_3

Prey, R. (2018). Nothing personal: algorithmic individuation on music streaming platforms. Media, Culture \& Society, 40(7), 1086-1100. https://doi.org/10.1177/0163443717745147

Prey, R. (2019). Background by Design: Listening in the Age of Streaming. Naxos Musicology International, $1(1)$.

Sloboda, J.A. (2010). Music in everyday life: The role of emotions. In P. N. Juslin \& J. A. Sloboda (Eds.), Handbook of music and emotion: Theory, research, applications. Oxford: Oxford University Press. https://doi. org/10.1093/acprof

SpotifyTechnology S.A. (2018). Form F-1 Registration Statement Unde the Securities Act of 1933 [Prospectus for IPO]. Retrieved January 20, 2020, from https://www.sec.gov/Archives/edgar/ data/1639920/000119312518063434/d494294df1.htm

Sterne, J. (1997). Sounds like the Mall of America: Programmed Music and the Architectonics of Commercial Space. Ethnomusicology, 41(1), 22. https://doi.org/10.2307/852577

Stockfelt, O. (1997). Adequate Modes of Listening. In D. Schwarz, A. Kassabian, \& L. Siegel (Eds.), Keeping Score: Music, Disciplinarity, Culture. Charlottesville: University Press of Virginia.

van Dijck, J. (2014). Datafication, dataism and dataveillance: Big data between scientific paradigm and ideology. Surveillance and Society, 12(2), 197-208. Retrieved from https://doi.org/10.24908/ss.v12i2.4776

Vonderau, P. (2019). The Spotify Effect: Digital Distribution and Financial Growth. Television \& New Media, 20(1), 3-19. https://doi.org/10.1177/1527476417741200

Werner, A. (2020). Organizing music, organizing gender: algorithmic culture and Spotify recommendations. Popular Communication, 18(1), 78-90. https://doi.org/10.1080/15405702.2020.1715980

Rasmus Rex Pedersen

Department of Communication and Arts

Roskilde University 